\title{
Kraftstoffe neu denken
}

Liebe Leserin, lieber Leser,

die individuelle Mobilität, so wie wir sie heute kennen und schätzen, verdanken wir nicht zuletzt Verbrennungsmotoren und Kraftstoffen, die wir überwiegend aus fossilen Ressourcen gewinnen. Doch bei der Verbrennung dieser fossilen Kraftstoffe entstehen unter anderem $\mathrm{CO}_{2}$-Emissionen, die unsere Luftqualität belasten.

Für Mensch und Tier ist ein ausgeglichener Kohlenstoffzyklus lebensnotwendig. Die Verbrennung fossiler Ressourcen bringt diesen Zyklus aus dem Gleichgewicht. Dieses Gleichgewicht können wir wieder herstellen, wenn wir die Ergebnisse des Klimagipfels in Paris umsetzen. Einer der Stellhebel dafür ist ein $\mathrm{CO}_{2}$-neutraler Verkehr. Mit Elektromobilität erreichen wir lokale Emissionsfreiheit. Elektromobilität ist aber kein ganzheitlicher Lösungsansatz. Weltweite Finanzierbarkeit, Langstrecken- und Schwerlastmobilität sind nicht sichergestellt.

Diese Lücke können $\mathrm{CO}_{2}$-neutrale Kraftstoffe mit ihrer hohen Energiedichte schließen. Besonders geeignet dafür sind synthetische Kraftstoffe. Wenn die Herstellung im großindustriellen Maßstab gelingt, können die fossilen Energieträger substituiert werden. Damit könnten wir sogar Bestandsfahrzeuge abdecken. Die Investitionen für die Produktentstehung und die Energieverteilung für den Verkehr sind weltweit vorhanden und können weiter genutzt werden. Synthetische Kraftstoffe können zudem gezielt designt werden. Damit haben sie das zusätzliche Potenzial einer rußfreien Verbrennung. Das wäre ein Durchbruch bei der Luftqualität. Erste Versuche sind vielversprechend.
Wir stehen aber erst am Anfang. Weitere Investitionen sind nötig, nicht nur in die Forschung, auch in die Herstellung dieser neuen Kraftstoffe. Gezielt designte synthetische Kraftstoffe geben uns die Chance, nicht nur bei einigen Millionen von Neufahrzeugen anzusetzen, sondern zusätzlich bei über einer Milliarde von Bestandsfahrzeugen ein Befreiungsschlag für die Branche. Neue Kraftstoffe und Elektromobilität können uns in eine nachhaltige, $\mathrm{CO}_{2}$ neutrale und emissionsfreie Mobilität führen. Nur beide Wege zusammen sind zielführend.

Wir haben in diesem Sonderheft wichtige Erkenntnisse und Aspekte der Kraftund Schmierstoffe der Zukunft zusammengetragen. Wir hoffen, damit neue Impulse geben zu können, und wünschen Ihnen viel Spaß bei der Lektüre.

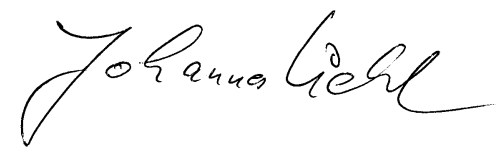

Dr. Johannes Liebl

Herausgeber ATZ I MTZ | ATZelektronik

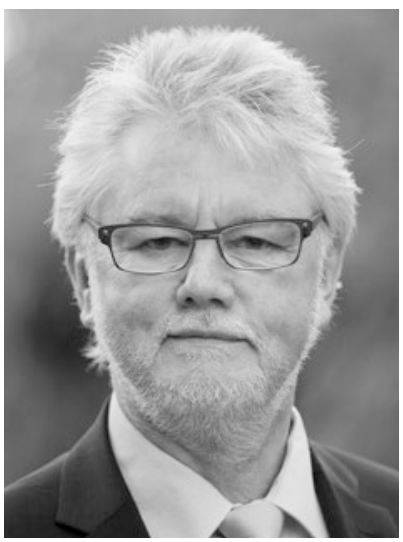

\section{UNABHÄNGIGE LABOREXPERTISE}

Das SGS Fuel Technology Centre ist im Automobilsektor sowie in der Petrochemie anerkannter Partner für Laboranalysen, Marktstudien und F\&E-Support.

Bekannt ist die SGS für ihre internationale Kraftstoffuntersuchungen, die seit mehr als 25 Jahren in über 190 Ländern laufen. Der Fuel Survey liefert der Industrie unverzichtbare Daten über Qualität, Risiken und Trends bei Kraftstoffen und Additiven.

Nutzen auch Sie unsere Services:

Daten aus dem

World Wide Fuel Survey

- Untersuchung von Kraftstoffqualitäten

- Analyse von Spezifikationsund Normparametern (u.a. DIN, ASTM)

- Kundenspezifische Analysen- und Methodenentwicklung

- Akkreditierte Untersuchung von Schmierölparametern

- Standardisierter Öl-Check von Schmierstoffen, Oil Condition Monitoring z. B. für Motoren sowie Getriebe in Fahrzeugen und stationären Anlagen

SGS Germany GmbH

Oil, Gas \& Chemical Services

$t+4030101-318$

de.ogc@sgs.com

www.sgsgroup.de

DIE SGS-GRUPPE IST DAS WELTWEIT FÜHRENDE UNTERNEHMEN IN DEN BEREICHEN PRÜFEN, TESTEN, VERIFIZIEREN UND ZERTIFIZIEREN. 